\title{
Density and pair correlation function of confined identical particles: The Bose-Einstein case
}

\author{
F. Brosens and J. T. Devreese* \\ Departement Natuurkunde, Universiteit Antwerpen (UIA), Universiteitsplein 1, B-2610 Antwerpen, Belgium \\ L. F. Lemmens \\ Departement Natuurkunde, Universiteit Antwerpen (RUCA), Groenenborgerlaan 171, B-2020 Antwerpen, Belgium
}

(Received 10 January 1997; revised manuscript received 7 March 1997)

\begin{abstract}
Two basic correlation functions are calculated for a model of $N$ harmonically interacting identical particles in a parabolic potential well. The density and the pair correlation function of the model are investigated for the boson case. The dependence of these static response properties on the complete range of the temperature and of the number of particles is obtained. The calculation technique is based on the path integral approach of symmetrized density matrices for identical particles in a parabolic confining well. [S1063-651X(97)14306-9]

PACS number(s): 05.30.-d, 03.75.Fi, 32.80.Pj.
\end{abstract}

\section{INTRODUCTION}

Generalizing the Feynman approach of identical particles in a box [1] to the case of identical particles in a parabolic confining potential, the present authors derived analytic expressions for the propagator and for the partition function of a system of $N$ harmonically interacting identical particles (bosons or fermions) in a parabolic well [2], hereafter referred to as I. This model, giving rise to repetitive Gaussian integrals, also allows us to obtain the generating function for the partition function. For an ideal gas of noninteracting particles in a parabolic well, this generating function coincides with the grand-canonical partition function. For interacting particles this generating function circumvents the constraints on the summation over the cycles of the permutation group at the expense of doing an extra path integral.

In the present paper the one- and two-point correlation functions of the model are calculated using their generating function as we did for the thermodynamic properties of the model. Also, here we have to introduce extra path integrals of Gaussian nature to facilitate the cyclic summations.

The one- and two-point correlation functions of the model are obtained for the boson case as well as for the fermion case. But in view of the recent interest in Bose-Einstein condensation in a trap [3-5], the explicit evaluation and the discussion of the results are restricted to the boson case in the present paper. The fermion case will be studied in a forthcoming paper.

In the case of distinguishable particles, the correlation functions play a key role in the variational approximation for path integrals $[6,7]$. This variational method can be reformulated for indistinguishable particles, and the knowledge of the one- and two-point correlation functions for harmonic trial actions is as crucial as it is for distinguishable particles. For any algorithmic approach to many-body diffusion [8-10] for interacting particles, the knowledge of the correlation functions of the model is very useful to test the actual implementations. Furthermore, the model provides an example of

\footnotetext{
*Also at Universiteit Antwerpen (RUCA) and Technische Universiteit Eindhoven, The Netherlands.
}

an exactly tractable system with interactions, which clearly exhibits the effects of Bose-Einstein condensation in the specific heat [11] and in the moment of inertia [12].

The one-body potential energy $V_{1}$ and the two-body potential energy $V_{2}$ of the model system are given by

$$
\begin{gathered}
V=V_{1}+V_{2}, \quad V_{1}=\frac{m \Omega^{2}}{2} \sum_{j=1}^{N} \mathbf{r}_{j}^{2}, \\
V_{2}=-\frac{m \omega^{2}}{4} \sum_{j, l=1}^{N}\left(\mathbf{r}_{j}-\mathbf{r}_{l}\right)^{2} .
\end{gathered}
$$

The two-body interaction is assumed to be repulsive; replacing $-\omega^{2}$ by $\omega^{2}$ in $V_{2}$ gives the attractive case. As a result of the diagonalization, one obtains in each dimension one degree of freedom (the center of mass) with frequency $\Omega$, and $N-1$ degrees of freedom with frequency $w$ given by

$$
w=\sqrt{\Omega^{2}-N \omega^{2}} .
$$

For this many-body system, distinguishability of the particles therefore implies that one is dealing with a system that reduces to $3 N$ degrees of freedom, each degree of freedom representing one linear harmonic oscillator. It is clear that for such a system the propagator, the thermodynamic functions, and the correlation functions are well known [13].

For identical particles (bosons or fermions) the propagator can be obtained from the decomposition of the underlying processes in terms of four orthogonal processes with welldefined boundary conditions [8-10]. A typical sample path for fermions is provided by the subprocess with absorption at the boundary for the $x$ direction (leading to a fermion diffusion process), while for the $y$ and $z$ directions a boson diffusion process with reflection at the boundary has to be used. This procedure is used to generate the trajectory of the walkers (including the $y$ and $z$ components). The path of a walker in this particular subprocess terminates if the motion in Euclidean time is absorbed along the $x$ direction. Indistinguishability therefore has the important effect of making the coupled oscillator problem in three dimensions a genuine three-dimensional (3D) problem. 
In I we showed how extra degrees of freedom for the center of mass can be introduced (in Fourier space), leading to a propagator which factorizes. The extra degrees of freedom can be integrated out afterwards. As a consequence, the internal degrees of freedom of the interacting oscillator system can be considered as the degrees of freedom of another noninteracting oscillator system. This mapping allows us to use the grand-canonical partition function of the noninteracting system as the generating function for the system in interaction, provided the fugacity and hence the thermodynamical potential are identified as usual.

The calculation of the density and the pair correlation function heavily relies on the calculations presented in I, which makes it difficult to make this paper self-contained without repeating some of the material presented in I. We tried to overcome this inconvenience partly by using the same notation as in I, and by a limited number of explicit references to the detailed manipulations in I if similar situations are encountered.

The paper is organized as follows. In Sec. II the one- and two-point correlation functions are calculated for identical particles (bosons or fermions). In Sec. III the density and the pair correlation function are analyzed for the boson case. In Sec. IV we discuss the results, and draw some conclusions.

\section{STATIC RESPONSE PROPERTIES OF A MANY-BODY SYSTEM}

For the calculation of the static response properties of a many-body system, the correlation functions $\Sigma_{l}\left\langle e^{i \mathbf{q} \cdot \mathbf{r}_{l}}\right\rangle_{I}$ and $\Sigma_{l, l}\left\langle e^{i \mathbf{q} \cdot\left(\mathbf{r}_{l}-\mathbf{r}_{l^{\prime}}\right)}\right\rangle_{I}$ are the key ingredients. The subscript $I$ emphasizes that identical particles are considered (which can be specified to be bosons with subscript $B$ or fermions with subscript $F$ ) in three-dimensions. In the path integral approach the expectation values of an expression $A\left(\overline{\mathbf{r}}^{\prime}, \tau\right)$ are given by

$$
\langle A(\tau)\rangle_{I}=\frac{\int d \overline{\mathbf{r}} \int d \overline{\mathbf{r}}^{\prime} K_{I}\left(\overline{\mathbf{r}}, \beta \mid \overline{\mathbf{r}}^{\prime}, \tau\right) A\left(\overline{\mathbf{r}}^{\prime}, \tau\right) K_{I}\left(\overline{\mathbf{r}}^{\prime}, \tau \mid \overline{\mathbf{r}}, 0\right)}{\int d \overline{\mathbf{r}} K_{I}(\overline{\mathbf{r}}, \beta \mid \overline{\mathbf{r}}, 0)},
$$

where $K_{I}$ is the statistical propagator of the identical particles and $\overline{\mathbf{r}}$ is the $3 N$-dimensional vector containing the coordinates $\mathbf{r}_{1}, \ldots, \mathbf{r}_{N}$ of the $N$ particles. In this notation the probability density, the pair correlation function and their Fourier transforms are given by

$$
\begin{aligned}
n(\mathbf{r})= & \frac{1}{N}\left\langle\sum_{l=1}^{N} \delta\left(\mathbf{r}-\mathbf{r}_{l}\right)\right\rangle_{I}=\int \frac{d \mathbf{q}}{(2 \pi)^{3}} n_{\mathbf{q}} e^{-i \mathbf{q} \cdot \mathbf{r}} \\
\leftrightarrow & n_{\mathbf{q}}=\frac{1}{N} \sum_{l=1}^{N}\left\langle e^{i \mathbf{q} \cdot \mathbf{r}_{l}}\right\rangle_{I},
\end{aligned}
$$

$$
\begin{aligned}
g(\mathbf{r})= & \frac{1}{N}\left\langle\sum_{l, l^{\prime} \neq l}^{N} \delta\left(\mathbf{r}-\mathbf{r}_{l}+\mathbf{r}_{l^{\prime}}\right)\right\rangle=\int \frac{d \mathbf{q}}{(2 \pi)^{3}} g_{\mathbf{q}} e^{-i \mathbf{q} \cdot \mathbf{r}} \\
& \leftrightarrow g_{\mathbf{q}}=\frac{1}{N} \sum_{l, l^{\prime} \neq l}^{N}\left\langle e^{i \mathbf{q} \cdot\left(\mathbf{r}_{l}-\mathbf{r}_{l^{\prime}}\right)}\right\rangle_{I} .
\end{aligned}
$$

Collecting the appropriate expressions for the propagators $K_{I}\left(\overline{\mathbf{r}}, \beta \mid \overline{\mathbf{r}}^{\prime}, \tau\right)$ and $K_{I}\left(\overline{\mathbf{r}}^{\prime}, \tau \mid \overline{\mathbf{r}}, 0\right)$ from I, one sees that the Fourier transforms $n_{\mathbf{q}}$ and $g_{\mathbf{q}}$ are given by

$$
\begin{aligned}
n_{\mathbf{q}}= & \frac{1}{N Z_{I}} \iint \frac{d \mathbf{R} d \mathbf{k}}{(2 \pi)^{3}} e^{i \mathbf{k} \cdot \mathbf{R}} \int d \overline{\mathbf{r}} e^{-i \overline{\mathbf{k}} \cdot \overline{\mathbf{r}}} \sum_{l} e^{i \mathbf{q} \cdot \mathbf{r}_{l}} \frac{1}{N !} \\
& \times \sum_{p} \xi^{p} \prod_{j=1}^{N} K\left((P \mathbf{r})_{j}, \beta \mid \mathbf{r}_{j}, 0\right)_{w}, \\
g_{\mathbf{q}}= & \frac{1}{N Z_{I}} \iint \frac{d \mathbf{R} d \mathbf{k}}{(2 \pi)^{3}} e^{i \mathbf{k} \cdot \mathbf{R}} \int d \overline{\mathbf{r}} e^{-i \overline{\mathbf{k}} \cdot \overline{\mathbf{r}}} \frac{1}{N !} \\
& \times \sum_{p} \xi^{p} \sum_{l, l^{\prime} \neq l}^{N} e^{i \mathbf{q} \cdot\left(\mathbf{r}_{l}-\mathbf{r}_{l^{\prime}}\right)} \prod_{j=1}^{N} K\left((P \mathbf{r})_{j}, \beta \mid \mathbf{r}_{j}, 0\right)_{w},
\end{aligned}
$$

where $Z_{I}$ is the partition function, $K\left(\mathbf{r}_{j}^{\prime}, \beta \mid \mathbf{r}_{j}, 0\right)_{w}$ is the propagator of a 3D harmonic oscillator with frequency $w$, $P$ denotes a permutation matrix, and $\xi=-1$ assures the required antisymmetry for fermions, whereas $\xi=+1$ describes bosons. We first show how a tractable expression can be obtained for $n_{\mathbf{q}}$, and subsequently use an analogous procedure to calculate $g_{\mathbf{q}}$.

\section{A. Single-particle expectation values}

Using the cyclic decomposition, and denoting by $M_{/}$the number of cycles of length $\ell$, the expression for $n_{\mathbf{q}}$ can be written in terms of the cycles in the same way as we did for the partition function in I:

$$
\begin{aligned}
n_{\mathbf{q}}= & \frac{1}{N Z_{I}} \iint \frac{d \mathbf{R} d \mathbf{k}}{(2 \pi)^{3}} e^{i \mathbf{k} \cdot \mathbf{R}} \sum_{M_{1} \cdots M_{N}} \sum \ell M_{\ell} \mathcal{K} /(\mathbf{k}, \mathbf{q}) \\
& \times \frac{\xi^{(\ell-1) M_{\ell}}}{M_{\ell^{\prime} ! \ell^{M_{\ell}}}(\mathcal{K} /(\mathbf{k}))^{M_{\ell^{\prime}-1}}} \\
& \times \prod_{\ell^{\prime} \neq \ell^{\prime}} \frac{\xi^{\left(\ell^{\prime}-1\right) M_{\ell^{\prime}}}}{M_{\ell^{\prime} !\left(\ell^{\prime}\right)^{M_{\ell^{\prime}}}}\left(\mathcal{K}_{\ell^{\prime}}(\mathbf{k})\right)^{M_{\ell^{\prime}}}}
\end{aligned}
$$

where

$$
\begin{aligned}
\mathcal{K}(\mathbf{k}, \mathbf{q})= & \int d \mathbf{r}_{\ell+1} \int d \mathbf{r}_{\ell} \cdots \int d \mathbf{r}_{1} \delta\left(\mathbf{r}_{\ell+1}-\mathbf{r}_{1}\right) e^{i \mathbf{q} \cdot \mathbf{r}_{1}} \\
& \times \prod_{j=1}^{\ell} K\left(\mathbf{r}_{j+1}, \beta \mid \mathbf{r}_{j}, 0\right)_{w} e^{-i(1 / N) \mathbf{k} \cdot \mathbf{r}_{j}}
\end{aligned}
$$

and $\mathcal{K}_{\ell}(\mathbf{k})=\mathcal{K}_{\ell}(\mathbf{k}, \mathbf{q}=0)$ is precisely the same function as found in Eq. (2.20) of I for the determination of the partition 
function. Both the integrations over $\mathbf{k}$ and $\mathbf{R}$ only involve Gaussian integrands, and one eventually finds, after some algebra,

$$
n_{\mathbf{q}}=\exp \left[-\frac{\hbar q^{2}}{4 m N}\left(\frac{\operatorname{coth} \frac{1}{2} \beta \hbar \Omega}{\Omega}-\frac{\operatorname{coth} \frac{1}{2} \beta \hbar w}{w}\right)\right] \tilde{n_{\mathbf{q}}} .
$$

The factor in front of $\tilde{n_{\mathbf{q}}}$ accounts for the center of mass, and obviously reduces to unity in the noninteracting case where $w=\Omega$. The factor $\tilde{n}_{\mathbf{q}}$ itself denotes the expectation value of $\Sigma_{l} e^{i \mathbf{q} \cdot \mathbf{r}_{l}}$ in the subspace of the relative coordinate system only with partition function $\mathbb{Z}_{I}(N)$ :

$$
\begin{aligned}
\tilde{n_{\mathbf{q}}=} & \frac{1}{N_{\mathbb{Z}_{I}(N)}} \sum_{M_{1} \cdots M_{N}}\left[\sum M_{\ell \ell}\right. \\
& \left.\times \exp \left(-\frac{\hbar q^{2} \operatorname{coth} \frac{1}{2} \ell \beta \hbar w}{4 m w}\right)\right] \\
& \times \prod_{\ell} \frac{\xi^{(\ell-1) M_{\ell}}}{M_{\ell} ! \ell^{M_{\ell}}}\left(\frac{1}{2 \sinh \frac{1}{2} \ell \beta \hbar w}\right)^{3 M_{\ell}} .
\end{aligned}
$$

In $\mathcal{K}_{\curlywedge}(\mathbf{k}, \mathbf{q})$, one recognizes the partition function (over a time interval $\ell \beta$ ) of a driven 3D harmonic oscillator with frequency $w$,

$$
\begin{gathered}
\mathcal{K}(\mathbf{k}, \mathbf{q})=\int d \mathbf{r} K(\mathbf{r}, \ell \beta \mid \mathbf{r}, 0)_{w} e^{-\int_{0}^{\ell \beta} d \tau \mathbf{f}_{\mathbf{q}}(\tau) \cdot \mathbf{r}(\tau),} \\
\mathbf{f}_{\mathbf{q}}(\tau)=i \frac{1}{N} \mathbf{k} \sum_{j=1}^{\ell-1} \delta(\tau-j \beta)+i\left(\frac{\mathbf{k}}{N}-\mathbf{q}\right) \delta(\tau),
\end{gathered}
$$

which is known $[1,6]$ in closed form:

$$
\begin{gathered}
\mathcal{K}_{\ell}(\mathbf{k}, \mathbf{q})=\frac{1}{\left(2 \sinh \frac{\ell \beta}{2} \hbar w\right)^{3}} e^{\Phi_{\mathbf{q}}}, \\
\Phi_{\mathbf{q}}=\frac{\hbar}{2} \int_{0}^{\ell \beta} d \tau \int_{0}^{\ell \beta} d \sigma \frac{\mathbf{f}_{\mathbf{q}}(\tau) \cdot \mathbf{f}_{\mathbf{q}}(\sigma)}{2 m w} \\
\quad \times \frac{\cosh \left(\frac{\ell \beta}{2}-|\tau-\sigma|\right) \hbar w}{\sinh \frac{1}{2} \ell \beta \hbar w} .
\end{gathered}
$$

The calculation of $\Phi_{\mathbf{q}}$, given $f_{\mathbf{q}}(\tau)$ as a sum of $\delta$ functions, is straightforward. The result is

$$
\begin{aligned}
\Phi_{\mathbf{q}}= & -\frac{\hbar}{4 m w}\left(\frac{\ell k^{2}}{N^{2}} \operatorname{coth} \frac{1}{2} \beta \hbar w-2 \frac{\mathbf{k} \cdot \mathbf{q}}{N} \operatorname{coth} \frac{1}{2} \beta \hbar w\right. \\
& \left.+q^{2} \operatorname{coth} \frac{1}{2} \ell \beta \hbar w\right),
\end{aligned}
$$

and, consequently,

$$
\begin{aligned}
\mathcal{K}_{\nearrow}(\mathbf{k}, \mathbf{q})= & \mathcal{K}_{\ell}(\mathbf{k}) \exp \left(\frac{\hbar \mathbf{k} \cdot \mathbf{q}}{2 N m w} \operatorname{coth} \frac{1}{2} \beta \hbar w\right. \\
& \left.-\frac{\hbar q^{2}}{4 m w} \operatorname{coth} \frac{1}{2} \ell \beta \hbar w\right),
\end{aligned}
$$

$$
\mathcal{K}_{\curlywedge}(\mathbf{k})=\frac{1}{\left(2 \sinh \frac{\ell \beta}{2} \hbar w\right)^{3}} \exp \left(-\frac{\hbar}{4 m w} \frac{\ell k^{2}}{N^{2}} \operatorname{coth} \frac{1}{2} \beta \hbar w\right)
$$

Introducing the generating function $\mathcal{G}_{1}(u, \mathbf{q})$ $=\sum_{N=0}^{\infty}\left[\mathbb{Z}_{I}(N) N \tilde{n_{\mathbf{q}}}\right] u^{N}$,

$$
\begin{aligned}
\mathcal{G}_{1}(u, \mathbf{q})= & \sum_{N=0}^{\infty} \sum_{M_{1} \cdots M_{N}}\left[\sum M_{\ell \ell}\right. \\
& \left.\times \exp \left(-\frac{\hbar q^{2} \operatorname{coth} \frac{1}{2} \ell \beta \hbar w}{4 m w}\right)\right] \\
& \times \prod \frac{1}{M_{\ell} !}\left[\frac{\xi^{(\ell-1)} u^{\ell}}{\ell\left(2 \sinh \frac{1}{2} \ell \beta \hbar w\right)^{3}}\right]^{M_{\ell}},
\end{aligned}
$$

the summations can be done:

$$
\mathcal{G}_{1}(u, \mathbf{q})=\Xi_{I}(u) \sum_{\ell=1}^{\infty} \frac{\xi^{\ell-1} \exp \left(-\frac{\hbar q^{2}}{4 m w} \operatorname{coth} \frac{1}{2} \ell \beta \hbar w\right)}{\left(2 \sinh \frac{1}{2} \ell \beta \hbar w\right)^{3}} u^{\ell}
$$

where $\Xi_{I}(u)=\Sigma_{N=0}^{\infty} Z_{I}(N) u^{N}$ is the generating function of the partition function $Z_{I}(N)$ of $N$ identical oscillators in the relative coordinate system, studied in I. Consequently

$$
\tilde{n_{\mathbf{q}}}=\frac{1}{N} \sum_{\ell=1}^{N} \frac{\xi^{\ell-1} \exp \left(-\frac{\hbar q^{2}}{4 m w} \operatorname{coth} \frac{1}{2} \ell \beta \hbar w\right)}{\left(2 \sinh \frac{1}{2} \ell \beta \hbar w\right)^{3}} \frac{\mathbb{Z}_{I}(N-\ell)}{\mathbb{Z}_{I}(N)} .
$$

Considering the limit $\mathbf{q} \rightarrow 0$, it should be noted that the sum rule $\widetilde{n}_{\mathbf{q}=0}=1$ is indeed satisfied.

\section{B. Two-particle expectation values}

Similarly to the treatment of the single-particle correlation function, the Fourier transform, which allows us to treat the center-of-mass coordinate as an independent degree of free 
dom, is introduced. The cyclic decomposition of the permutations implies that a factor $e^{i \mathbf{q} \cdot \mathbf{r}_{l}}$ occurs once in each position of each cycle. Furthermore a different factor $e^{i \mathbf{q} \cdot \mathbf{r}_{l^{\prime}}}$ occurs in each position of each cycle which differs from $\mathbf{r}_{l}$ (i.e., the case $l=l^{\prime}$ has to be excluded if $\mathbf{r}_{l}$ and $\mathbf{r}_{l^{\prime}}$ are within the same cycle). Taking these bookkeeping considerations into account, the cyclic decomposition of the summation over the permutations leads to

$$
\begin{aligned}
& g_{\mathbf{q}}=\frac{1}{Z_{I} N} \iint \frac{d \mathbf{R} d \mathbf{k}}{(2 \pi)^{3}} e^{i \mathbf{k} \cdot \mathbf{R}} \sum_{M_{1} \cdots M_{N}}\left(\prod \frac{\xi^{(\ell-1) M_{\ell}}}{M_{\ell} ! \ell^{M_{\ell}}}\right) \\
& \times \sum M_{\ell}\left(\begin{array}{l}
\sum_{j=1}^{\ell} \mathcal{K}_{\ell}(\mathbf{k}, \mathbf{q} ; j+1)\left(\mathcal{K}_{\ell}(\mathbf{k})\right)^{M_{\ell^{\prime}}-1} \prod_{\ell^{\prime} \neq \ell^{\prime}}\left(\mathcal{K}_{\ell^{\prime}}(\mathbf{k})\right)^{M_{\ell^{\prime}}} \\
+\ell\left(M_{\ell}-1\right) \mathcal{K}_{\ell}(\mathbf{k}, \mathbf{q}) \mathcal{K}_{\ell}(\mathbf{k},-\mathbf{q})\left(\mathcal{K}_{\ell}(\mathbf{k})\right)^{M_{\ell^{\prime}}-2} \prod_{\ell^{\prime} \neq \ell^{\prime}}\left(\mathcal{K}_{\ell^{\prime}}(\mathbf{k})\right)^{M_{\ell^{\prime}}} \\
+\sum_{\ell^{\prime} \neq \ell^{\prime}} \ell^{\prime} M_{\ell^{\prime}} \mathcal{K}_{\ell}(\mathbf{k}, \mathbf{q}) \mathcal{K}_{\ell^{\prime}}(\mathbf{k},-\mathbf{q})\left(\mathcal{K}_{\ell}(\vec{k})\right)^{M_{\ell^{\prime}}-1}\left(\mathcal{K}_{\ell}(\mathbf{k})\right)^{M_{\ell^{\prime}-1}} \prod_{\ell^{\prime \prime} \neq \ell_{\ell} \ell^{\prime}}\left(\mathcal{K}_{\ell^{\prime \prime}}(\mathbf{k})\right)^{M_{\ell^{\prime \prime}}}
\end{array}\right),
\end{aligned}
$$

where $\mathcal{K}_{\curlywedge}(\mathbf{k}, \mathbf{q})$ and $\mathcal{K}_{\ell}(\mathbf{k})$ are defined as Sec. II A and a function $\mathcal{K}_{\curlywedge}(\mathbf{k}, \mathbf{q} ; j)$ is introduced which is given by

$$
\begin{aligned}
\mathcal{K}_{\curlywedge}(\mathbf{k}, \mathbf{q} ; j)= & \int d \mathbf{r}_{\ell+1} \int d \mathbf{r}_{\ell} \cdots \int d \mathbf{r}_{1} \delta\left(\mathbf{r}_{\ell+1}-\mathbf{r}_{1}\right) \\
& \times e^{i \mathbf{q} \cdot \mathbf{r}_{1}} e^{-i \mathbf{q} \cdot \mathbf{r}_{j}} \\
& \times \prod_{j^{\prime}=1}^{\ell} K\left(\mathbf{r}_{j^{\prime}+1}, \beta \mid \mathbf{r}_{j^{\prime}}, 0\right)_{w} \\
& \times e^{-i(1 / N) \mathbf{k} \cdot \mathbf{r}_{j^{\prime}} .}
\end{aligned}
$$

In $\mathcal{K} /(\mathbf{k}, \mathbf{q} ; j)$, one recognizes the partition function (over a time interval $\ell \beta$ ) of a driven 3D harmonic oscillator with frequency $w$ :

$$
\begin{aligned}
& \mathcal{K}_{\curlywedge}(\mathbf{k}, \mathbf{q} ; j+1)=\int d \mathbf{r} K(\mathbf{r}, \ell \beta \mid \mathbf{r}, 0)_{w} e^{-\int_{0}^{\ell \beta} d \tau \mathbf{h}_{\mathbf{q}}(\tau, j) \cdot \mathbf{r}(\tau)}, \\
& \mathbf{h}_{\mathbf{q}}(\tau, j)=i \frac{1}{N} \mathbf{k} \sum_{j^{\prime}=0}^{\ell-1} \delta\left(\tau-j^{\prime} \beta\right)-i \mathbf{q} \delta(\tau)+i \mathbf{q} \delta(\tau-j \beta) .
\end{aligned}
$$

Similarly as for single-particle correlation functions, this expression is known $[1,6]$ in closed form:

$$
\begin{aligned}
\mathcal{K}_{\ell}(\mathbf{k}, \mathbf{q} ; j+1) & =\left(\frac{1}{2 \sinh \frac{\ell \beta}{2} \hbar w}\right)^{3} e^{\Psi_{\mathbf{q}}(j)}, \\
\Psi_{\mathbf{q}}(j)= & \frac{\hbar}{2} \int_{0}^{\ell \beta} d \tau \int_{0}^{\ell \beta} d \sigma f \frac{\mathbf{h}_{\mathbf{q}}(\tau, j) \cdot \mathbf{h}_{\mathbf{q}}(\tau, j)}{2 m w} \\
& \times \frac{\cosh \left(\frac{\ell \beta}{2}-|\tau-\sigma|\right) \hbar w}{\sinh \frac{1}{2} \ell \beta \hbar w} .
\end{aligned}
$$

The explicit evaluation of the influence function $\Psi_{\mathbf{q}}(j)$ is somewhat involved but straightforward,

$$
\begin{aligned}
\Psi_{\mathbf{q}}(j)= & -\frac{\ell}{N^{2}} \frac{\hbar \mathbf{k}^{2}}{4 m w} \frac{e^{\beta \hbar w}+1}{e^{\beta \hbar w}-1} \\
& -\frac{\hbar \mathbf{q}^{2}}{2 m w} \frac{\cosh \frac{1}{2} \ell \beta \hbar w-\cosh \left(\frac{1}{2} \ell-j\right) \beta \hbar w}{\sinh ^{\frac{1}{2} \ell \beta \hbar w}},
\end{aligned}
$$

and, consequently,

$$
\mathcal{K}_{\ell}(\mathbf{k}, \mathbf{q} ; j+1)=\mathcal{K},(\mathbf{k}) \exp \left(-\frac{\hbar q^{2}}{2 m w} \frac{\cosh \frac{1}{2} \ell \beta \hbar w-\cosh \left(\frac{1}{2} \ell-j\right) \beta \hbar w}{\sinh \frac{1}{2} \ell \beta \hbar w}\right) .
$$

Using the results obtained in Sec. II A for $\mathcal{K}_{\curlywedge}(\mathbf{k}, \mathbf{q})$ and $\mathcal{K}_{\curlywedge}(\mathbf{k})$, the Fourier transform of the pair correlation function becomes 


$$
\begin{aligned}
& g_{\mathbf{q}}=\frac{1}{Z_{I} N} \iint \frac{d \mathbf{R} d \mathbf{k}}{(2 \pi)^{3}} e^{i \mathbf{k} \cdot \mathbf{R}} \\
& \times \sum_{M_{1} \cdots M_{N}}\left(\prod_{\ell} \frac{\xi^{(\ell-1) M_{\ell}}}{M_{\ell} ! \ell^{M_{\ell}}}\right) \prod(\mathcal{K} /(\mathbf{k}))^{M_{\ell}} \sum \ell M_{\ell}\left(\begin{array}{l}
\sum_{j=1}^{\ell} \exp \left(-\frac{\hbar q^{2}}{2 m w} \frac{\cosh \frac{1}{2} \ell \beta \hbar w-\cosh \left(\frac{1}{2} \ell-j\right) \beta \hbar w}{\sinh \frac{1}{2} \ell \beta \hbar w}\right) \\
+\ell\left(M_{\ell}-1\right) \exp \left(-\frac{1}{2} \frac{\hbar q^{2}}{m w} \operatorname{coth} \frac{1}{2} \ell \beta \hbar w\right) \\
+\sum_{\ell^{\prime} \neq \ell} \ell^{\prime} M_{\ell^{\prime}} \exp \left[-\frac{1}{4} \frac{\hbar q^{2}}{m w}\left(\operatorname{coth} \frac{1}{2} \ell \beta \hbar w+\operatorname{coth} \frac{1}{2} \ell^{\prime} \beta \hbar w\right)\right]
\end{array}\right) .
\end{aligned}
$$

The condition $\Sigma / \ell M_{\ell}=N$ on the cyclic decompositions simplifies $\Pi_{\ell}(\mathcal{K} /(\mathbf{k}))^{M /}$, and the integrations over $\mathbf{k}$ and $\mathbf{R}$ are then straightforward, resulting in $\left(\sinh \frac{1}{2} \beta \hbar w /\right.$ $\left.\sinh \frac{1}{2} \beta \hbar \Omega\right)^{3}$. The remaining summation over the cycles can again be done if one introduces the appropriate generating function

$$
\mathcal{G}_{2}(u, \mathbf{q})=\sum_{N=0}^{\infty}\left[Z_{I}(N) N g_{\mathbf{q}}\right] u^{N},
$$

which lifts the restriction on the number of cycles of given length. The summation is straightforward. With $b=e^{-\beta \hbar w}$, one obtains

$$
\begin{aligned}
\mathcal{G}_{2}(u, \mathbf{q})= & \Xi_{I}(u)\left\{\sum_{\ell=1}^{\infty} \frac{\xi^{\ell-1} u^{\ell} b^{\frac{3}{2} \ell}}{\left(1-b^{\ell}\right)^{3}}\right. \\
& \times \sum_{j=1}^{\ell-1} \exp \left(-\frac{\hbar q^{2}}{2 m w} \frac{\left(1-b^{j}\right)\left(1-b^{\ell-j}\right)}{1-b^{\ell}}\right) \\
& \left.+\left[\sum_{\ell=1}^{\infty} \frac{\xi^{\ell-1} u^{\ell} b^{(3 / 2) \ell}}{\left(1-b^{\ell}\right)^{3}} \exp \left(-\frac{\hbar q^{2}}{4 m w} \frac{1+b^{\ell}}{1-b^{\ell}}\right)\right]^{2}\right\} .
\end{aligned}
$$

Using $\left(\sum_{\ell=1}^{\infty} a_{\ell}\right)^{2}=\sum_{\ell=2}^{\infty} \sum_{j=1}^{\ell-1} a_{j} a_{\ell-j}$, and defining

$$
Q_{\ell, j}(b)=\frac{1-b^{\ell}}{\left(1-b^{j}\right)\left(1-b^{\ell-j}\right)},
$$

the terms can be combined into

$$
\begin{aligned}
\mathcal{G}_{2}(u, \mathbf{q})= & \Xi_{I}(u) \sum_{\ell=2}^{\infty} \frac{\xi^{\ell-1} u^{\ell} b^{(3 / 2) \ell}}{\left(1-b^{\ell}\right)^{3}} \\
& \times \sum_{j=1}^{\ell-1}\left[\exp \left(-\frac{\hbar q^{2}}{2 m w} \frac{1}{Q_{\ell, j}(b)}\right)\right. \\
& \left.+\xi\left(Q_{\ell, j}(b)\right)^{3} \exp \left(-\frac{\hbar q^{2}}{2 m w} Q_{\ell, j}(b)\right)\right] .
\end{aligned}
$$

It should be noticed that only cycles with length at least two contribute to the pair correlation function, as is to be expected. Because the series expansion of $\mathcal{G}_{2}(u, \mathbf{q})$ in powers of $u$ yields $\mathbb{Z}_{I}(N) N g_{\mathbf{q}}$ as the coefficient of $u^{N}$, one immediately obtains

$$
\begin{aligned}
N g_{\mathbf{q}}= & \frac{1}{\mathbb{Z}_{I}(N)} \sum_{\ell=2}^{N} Z_{I}(N-\ell) \frac{\xi^{\ell-1} b^{\frac{3}{2} \ell}}{\left(1-b^{\prime}\right)^{3}} \\
& \times \sum_{j=1}^{\ell-1}\left[\exp \left(-\frac{\hbar q^{2}}{2 m w} \frac{1}{Q_{\ell, j}(b)}\right)\right. \\
& \left.+\xi\left(Q_{\ell, j}(b)\right)^{3} \exp \left(-\frac{\hbar q^{2}}{2 m w} Q_{\ell, j}(b)\right)\right] .
\end{aligned}
$$

In the case of the pair correlation function $g_{\mathbf{q}}$, the sum rule $g_{\mathbf{q}=0}=N-1$ can also be checked. The proof proceeds by induction, but it is rather tedious and is omitted here. All details of this calculation are provided upon request.

\section{AND PAIR CORRELATION FUNCTION}

In this section the density and the pair correlation of the model are evaluated for the boson case, and the condensation effects on these quantities are studied.

\section{A. Density}

The density $n(r)$ in the case of boson statistics can be obtained from $n_{\mathbf{q}}$, and reads

$$
\begin{aligned}
n(r)= & \frac{1}{N} \sum_{\ell=1}^{N} \frac{Z_{I}(N-\ell)}{Z_{I}(N)} \frac{\xi^{\ell-1} b^{(3 / 2) \ell}}{\left(1-b^{\ell}\right)^{3}}\left(\frac{w m}{\pi \hbar} A_{\ell}\right)^{3 / 2} \\
& \times \exp \left(-\frac{m w r^{2}}{\hbar} A_{\ell}\right),
\end{aligned}
$$

with

$$
A_{\ell}=\frac{1}{\operatorname{coth} \frac{1}{2} \ell \beta \hbar w+\frac{1}{N}\left(\frac{w}{\Omega} \operatorname{coth} \frac{1}{2} \beta \hbar \Omega-\operatorname{coth} \frac{1}{2} \beta \hbar w\right)},
$$

where $r$ stands for the distance from the center of the confining potential. The density is centrosymmetric, as a conse- 


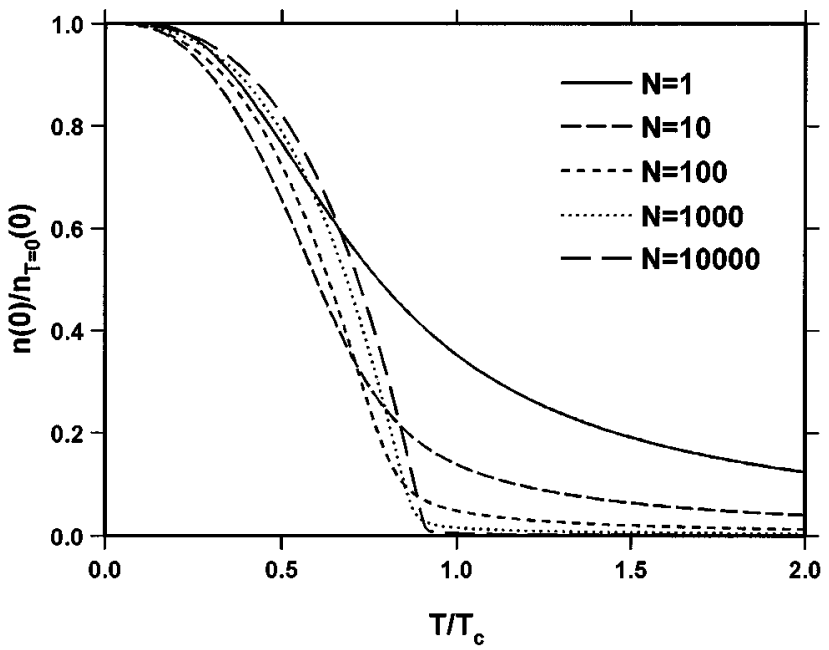

FIG. 1. Boson density $n(0) / n_{T=0}(0)$ at the origin as a function of temperature.

quence of the isotropy of the model. Introducing the activity $\rho_{j}$ as in I from $\mathbb{Z}_{B}(N)=\left[b^{3 / 2 N} / \Pi_{j=1}^{N}\left(1-b^{j}\right)^{3}\right] \Pi_{j=0}^{N} \rho_{j}$ the density can be rewritten as follows:

$$
\begin{aligned}
n(r)= & \frac{1}{N} \sum_{\ell=1}^{N} \frac{1}{\left(1-b^{\ell}\right)^{3}}\left(\frac{w m A_{\ell}}{\pi \hbar}\right)^{3 / 2} \exp \left(-\frac{m w}{\hbar} r^{2} A_{\ell}\right) \\
& \times \prod_{j=N-\ell+1}^{N} \frac{\left(1-b^{j}\right)^{3}}{\rho_{j}},
\end{aligned}
$$

which allows for a recursively defined expression well suited for numerical evaluation

$$
\begin{aligned}
n(r)= & \frac{1}{N} \frac{\left(1-b^{N}\right)^{3}}{\rho_{N}}\left(a_{1}+\frac{\left(1-b^{N-1}\right)^{3}}{\rho_{N-1}}\left\{a_{2}+\frac{\left(1-b^{N-2}\right)^{3}}{\rho_{N-2}}\right.\right. \\
& \left.\left.\times\left[a_{3}+\cdots+\frac{\left(1-b^{2}\right)^{3}}{\rho_{2}}\left(a_{N-1}+\frac{(1-b)^{3}}{\rho_{1}} a_{N}\right)\right]\right\}\right),
\end{aligned}
$$

with

$$
a_{\ell}=\left(\frac{w m}{\pi \hbar}\right)^{3 / 2} \frac{A_{\ell}^{3 / 2}}{\left(1-b^{\prime}\right)^{3}} e^{-\rho^{2} A_{\ell},}
$$

where $\rho=r \sqrt{m w / \hbar}$ is a natural dimensionless quantity proportional to the distance from the center. Since $T=t T_{c} \sim t N^{1 / 3}$ (where $T_{c}$ is the condensation temperature for the Bose-Einstein transition), $\rho / N^{1 / 6}$ is a natural quantity against which to plot the density. The results are summarized in two figures. In Fig. 1, the density $n(0) / n_{T=0}(0)$ in the origin is shown [where $n_{T=0}(0)$ is the density in the origin at zero temperature], and exhibits a pronounced dependence on the condensation temperature. In Fig. 2, $n(r) / n(0)$ is plotted as a function of $r$ for 1000 particles. For comparison, the corresponding densities for the case of distinguishable particles are plotted in Fig. 3. $T_{c}$ is only used as a reference temperature for comparison purposes to Fig. 2; it does not have the meaning of a condensation temperature if the particles are distinguishable.

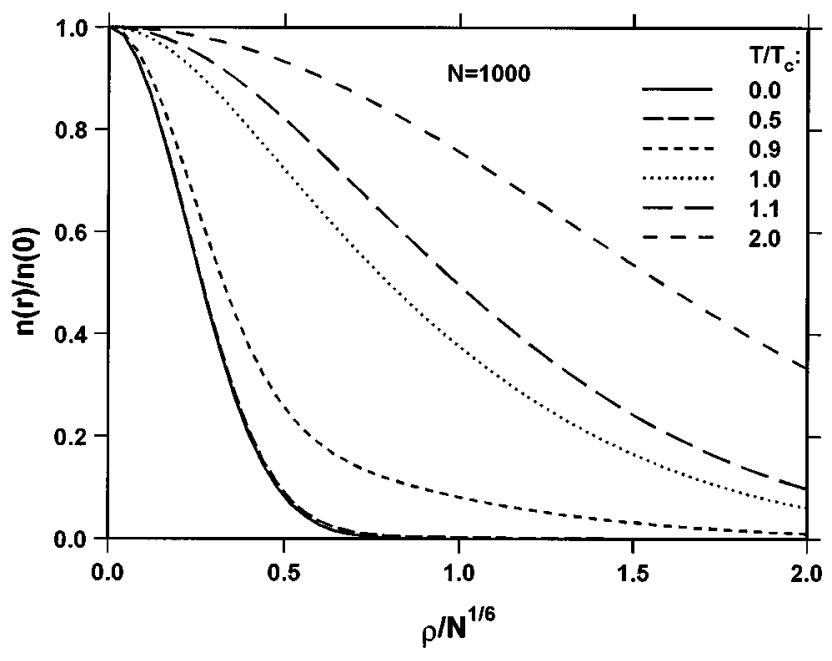

FIG. 2. Scaled density of bosons $n(r) / n(0)$ for 1000 particles as a function of the distance $r$ from the center for several temperatures.

The typical shape of the density as a function of the temperature is shown in Fig. 4 for $T=0, T=0.9 T_{c}, T=T_{c}$, and $T=1.1 T_{c}$, where the spatial dependence of the density $n(x, 0, z)$ is plotted at a fixed value $y=0$ for 1000 particles. It should be noted that the sudden appearance of an intense peak below $T_{c}$ when sweeping through the condensation temperature is also manifestly present in isotropic systems.

The center-of-mass contribution to the density can be substantial for a limited number of particles. For 1000 particles this single degree of freedom quantitatively makes a negligible contribution to the density as a function of $r \sqrt{w}$; the effects of the interaction enter in the eigenfrequency $w=\sqrt{\Omega^{2}-N \omega^{2}}$, which determines the scaling parameters in the figures.

\section{B. Pair correlation function}

An analogous analysis to that for the density can be made for the pair correlation function:

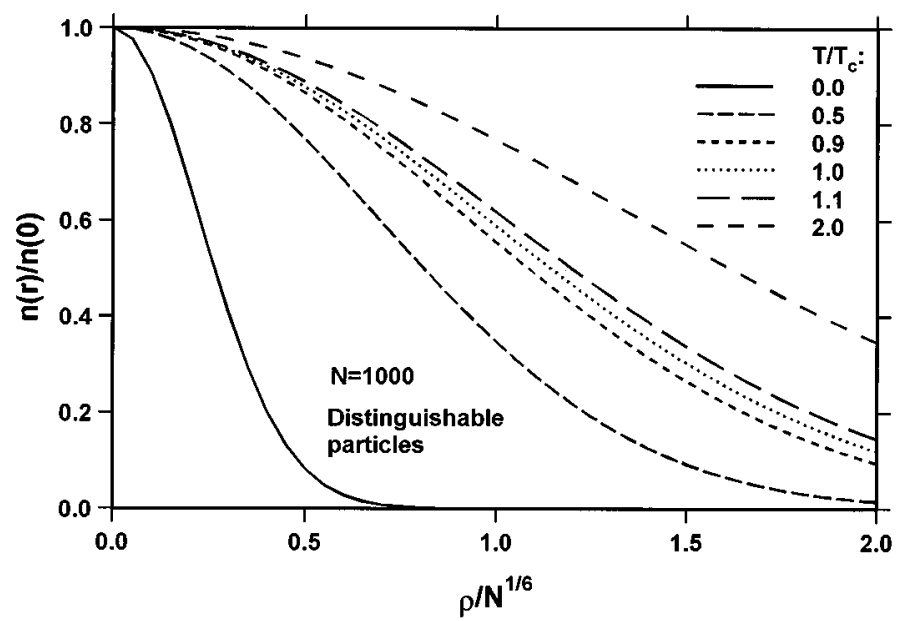

FIG. 3. Scaled density $n(r) / n(0)$ for 1000 distinguishable particles for comparison to Fig. 2. 

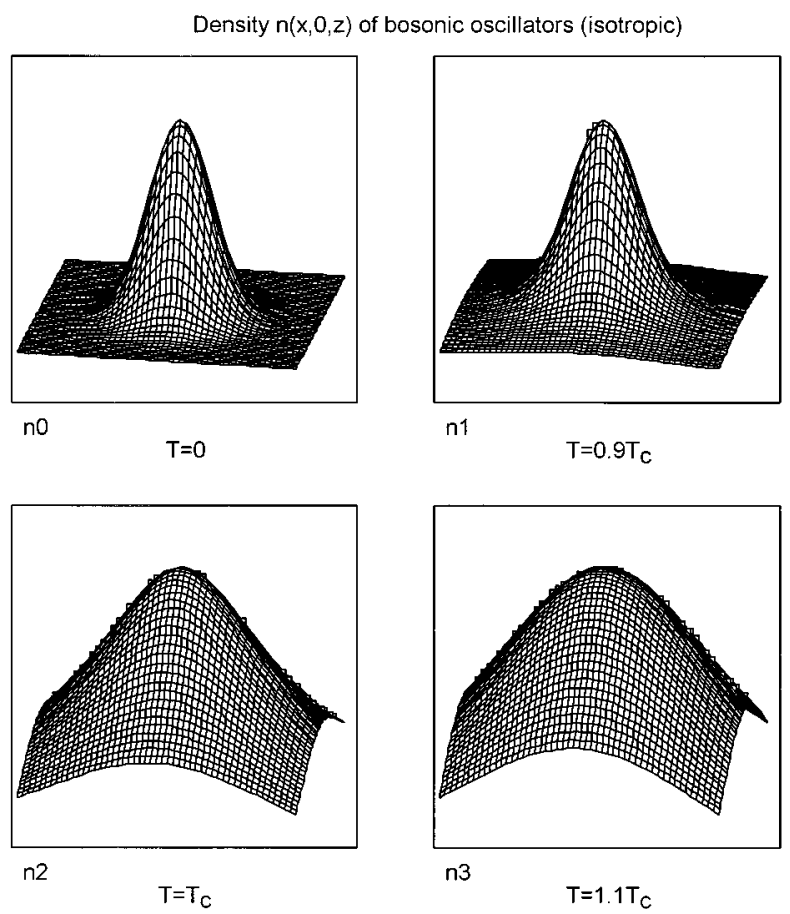

FIG. 4. Probability density $n(x, 0, z)$ for 1000 particles as a function of $x$ and $z$ for $T=0, T=0.9 T_{c}, T=T_{c}$, and $T=1.1 T_{c}$.

$$
\begin{aligned}
g(r)= & \frac{1}{N} \sum_{\ell=2}^{N} \frac{Z_{I}(N-\ell)}{\mathbb{Z}_{I}(N)} \frac{\xi^{\ell-1} b^{(3 / 2) \ell}}{\left(1-b^{\ell}\right)^{3}} \\
& \times \sum_{j=1}^{\ell-1}\left(\frac{m w}{2 \pi \hbar} Q_{\ell, j}\right)^{3 / 2}\left[\exp \left(-\frac{m w r^{2}}{2 \hbar} Q_{\ell, j}\right)\right. \\
& \left.+\xi \exp \left(-\frac{m w r^{2}}{2 \hbar} \frac{1}{Q_{\ell, j}}\right)\right] .
\end{aligned}
$$

Introducing

$$
\begin{aligned}
q_{\ell}= & \sum_{j=1}^{\ell-1}\left(\frac{m w}{2 \pi \hbar} Q_{\ell, j}\right)^{3 / 2} \frac{1}{\left(1-b^{\ell}\right)^{3}}\left[\exp \left(-\frac{m w r^{2}}{2 \hbar} Q_{\ell, j}\right)\right. \\
& \left.+\exp \left(-\frac{m w r^{2}}{2 \hbar} \frac{1}{Q_{\ell, j}}\right)\right]
\end{aligned}
$$

(with $q_{1}=0$ ), a recurrence relation for $g(r)$ can be obtained similarly as for $n(r)$, but with $a_{\ell}$ from Eq. (3.5) replaced by $q_{\ell}$. In the same units as for the density, $g(r) / g(0)$ is plotted in Fig. 5. Similarly as for the density in the Sec. III A $g(r) / g(0)$ of distinguishable particles is shown in Fig. 6 for comparison to the boson case in Fig. 5.

The interpretation of Fig. 5 requires some caution, because $g(r) / g(0)$ is plotted, and the magnitude of $g(0)$ strongly depends on the condensation temperature. Nevertheless, it is clearly seen that the probability of finding another particle at a relatively small distance $r$ from some particle is very pronounced in the condensate. Above the critical temperature a more substantial contribution is obtained at relatively large distances, but the boson character still manifests itself by a larger probability of finding particles at relatively short distances from the center.

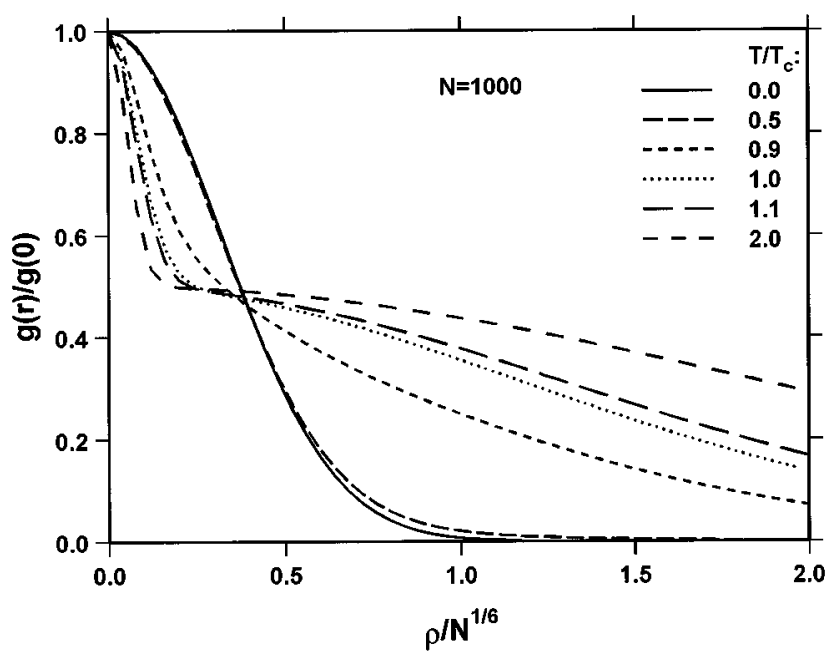

FIG. 5. Scaled pair correlation function $g(r) / g(0)$ of 1000 bosons as a function of the distance $r$ for several temperatures.

\section{DISCUSSION AND CONCLUSIONS}

The present paper concludes the boson part of a study of an exactly soluble model containing one type of particles. The system exhibits condensation at a finite temperature $T_{c}$. Thermodynamical quantities such as the internal energy, the specific heat, and the moment of inertia have been studied before by the present authors, and also by others [14$18]$, as far as some noninteracting aspects or ground state properties [19] are concerned. The density of this model is an important response property. Precisely the concentration variations as a function of the cooling and the field are invoked to establish the condensation transition $[3,4]$. Therefore it is comforting that the predictions of this theoretical model-which of course constitutes a simplification-bare some resemblance to the simulated density of an anisotropic boson oscillator model [20], and to the experimental situation in several aspects. It should be noted (i) that the magnetically induced anisotropy of the trap is not taken into account in the present paper, and (ii) that the interparticle interactions are replaced by harmonic two-body interactions.

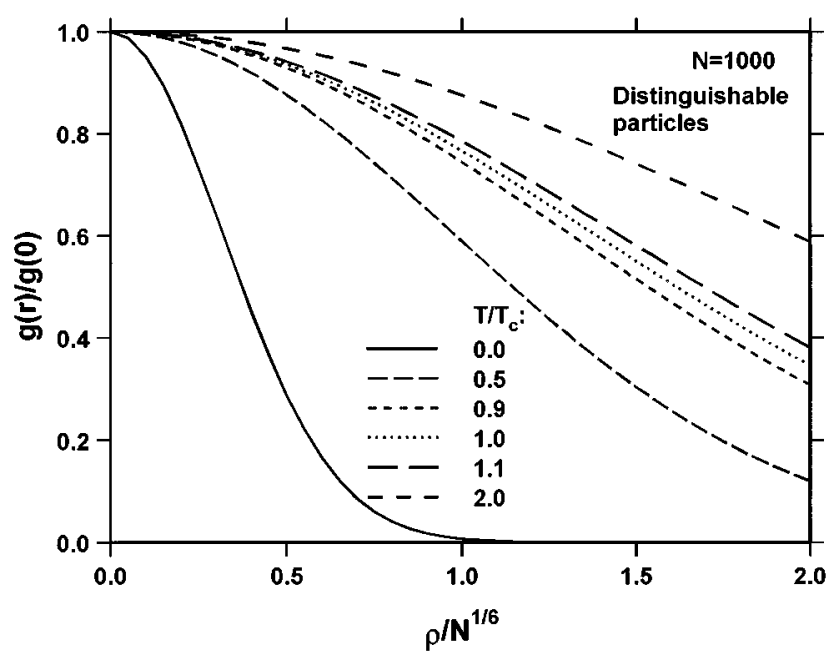

FIG. 6. Scaled pair correlation function $g(r) / g(0)$ of 1000 distinguishable particles for comparison to Fig. 5 . 
Furthermore it is assumed that the time scale of the experiment allows for an interpretation in terms of the thermal equilibrium response properties. It is clear that these simplifications deserve further investigations.

In addition, the pair correlation function of the model is an important quantity especially if one wants to investigate the modifications due to a more realistic interparticle interaction using a variational approach. Indeed, an estimate of the effective interparticle potential along the lines of Refs. $[6,7]$ requires the pair correlation function of this exactly soluble model, that is taken as the zeroth-order approximation to the system. From the methodological point of view, the projection and the generating function technique allows one to obtain tractable and exact expressions for the thermo- dynamic quantities and for the static response functions of a system of identical boson oscillators.

\section{ACKNOWLEDGMENTS}

Part of this work was performed in the framework of the FWO Project Nos. 2.0093.91, 2.0110.91, G. 0287.95 and WO.073.94N (Wetenschappelijke Onderzoeksgemeenschap, Scientific Research Community of the FWO on "LowDimensional Systems', ), and in the framework of the European Community Program Human Capital and Mobility through Contract Nos. CHRX-CT93-0337 and CHRX-CT930124. One of the authors (F.B.) acknowledges the FWO (Fund for Scientific Research-Flanders) for financial support.
[1] R. P. Feynman, Statistical Mechanics, a Set of Lectures (Benjamin Inc., Reading, MA, 1972).

[2] F. Brosens, J. T. Devreese, and L. F. Lemmens, Phys. Rev. E 55, 227 (1992).

[3] M. H. Anderson, J. R. Ensher, M. R. Matthews, C. E. Wieman, and E. A. Cornell, Science 269, 198 (1995).

[4] K. B. Davis, M. O. Mewes, M. R. Andrews, N. J. van Druten, D. S. Durfee, D. M. Kurn, and W. Ketterle, Phys. Rev. Lett. 75, 3969 (1995).

[5] C. C. Bradley, C. A. Sackett, J. J. Tollett, and R. G. Hulet, Phys. Rev. Lett. 75, 1687 (1995).

[6] R. P. Feynman and A. R. Hibbs, Quantum Mechanics and Path Integrals (McGraw-Hill, New York, 1965).

[7] R. P. Feynman and H. Kleinert, Phys. Rev. A 34, 5080 (1986).

[8] L. F. Lemmens, F. Brosens, and J. T. Devreese, Phys. Lett. A 189, 437 (1994).

[9] F. Brosens, J. T. Devreese, and L. F. Lemmens, Solid State Commun. 96, 137 (1995).

[10] L. F. Lemmens, F. Brosens, and J. T. Devreese, Phys. Rev. E
53, 4467 (1996).

[11] F. Brosens, J. T. Devreese, and L. F. Lemmens, Solid State Commun. 100, 123 (1996).

[12] F. Brosens, J. T. Devreese, and L. F. Lemmens, Phys. Rev. A 55, 2453 (1997).

[13] G. W. Ford, M. Kac, and P. Mazur, J. Math. Phys. 6, 504 (1965), represented in Mathematical Physics in One Dimension, edited by E. H Lieb and D. C. Mattis (Academic, New York, 1966).

[14] S. Grossman and M. Holthaus, Z. Naturforsch. 50a, 323; 921 (1995).

[15] S. Grossman and M. Holthaus, Phys. Lett. A 208, 188 (1995).

[16] W. Ketterle and N. J. van Druten, Phys. Rev. A 54, 656 (1996).

[17] K. Kirsten and D. J. Toms, Phys. Rev. A 54, 4188 (1996).

[18] H. Haugerud, T. Haugset, and F. Ravndal, Phys. Lett. A 225, 18 (1997).

[19] L. Cohen and C. Lee, J. Math. Phys. 26, 3105 (1985).

[20] W. Krauth, Phys. Rev. Lett. 77, 3695 (1996). 